\title{
Quantitative and Probabilistic Modeling in Pathway Logic
}

\author{
Alessandro Abate \\ Yu Bai \\ Natalie Sznajder \\ Carolyn Talcott \\ Ashish Tiwari
}

Electrical Engineering and Computer Sciences University of California at Berkeley

Technical Report No. UCB/EECS-2006-180

http://www.eecs.berkeley.edu/Pubs/TechRpts/2006/EECS-2006-180.html

December 16, 2006 
Copyright @ 2006, by the author(s).

All rights reserved.

Permission to make digital or hard copies of all or part of this work for personal or classroom use is granted without fee provided that copies are not made or distributed for profit or commercial advantage and that copies bear this notice and the full citation on the first page. To copy otherwise, to republish, to post on servers or to redistribute to lists, requires prior specific permission. 


\title{
Quantitative and Probabilistic Modeling in Pathway Logic
}

\author{
A. Abate,${ }^{1,2}$, Y. Bai,${ }^{1,3}$, N. Sznajder, ${ }^{1,4}$, C. Talcott,${ }^{1}$, and A. Tiwari ${ }^{1}$ \\ 1 Computer Sciences Laboratory, SRI International \{clt,tiwari@csl.sri.com\} \\ 2 Department of Electrical Engineering and Computer Sciences, University of \\ California, at Berkeley \{aabate@eecs.berkeley.edu\} \\ 3 Biophysics Program, Stanford University \{yubai@stanford.edu \\ 4 Ecole Normale Superiéure \{sznajder@clipper.ens.fr\}
}

\begin{abstract}
This paper presents a study of possible extensions of Pathway Logic to represent and reason about semiquantitative and probabilistic aspects of biological processes. The underlying theme is annotation of reaction rules with affinity information that can be used in different simulation strategies. Several such strategies were implemented, and experiments carried out to test feasibility, and to compare results of different approaches. Dimerization in the ErbB signalling network, important in cancer biology, was used as a test case.
\end{abstract}

\section{Introduction}

Biological networks have complex interconnections, non-linear responses to stimuli and self-regulation. This presents clear challenges for modeling and studying behaviors of such networks, and it is important to efficiently organize and represent knowledge about biological networks needed for modeling. Pathway Logic $[1-3]$ is an approach to modeling cellular processes based on symbolic logic. It allows one to model aspects of the structure and state of interacting components, to represent individual process steps (reactions) and to study possible ways a system could evolve using techniques based on logical inference. Reactions can be modeled at many levels of detail ranging from micro steps representing events such as phosphorlyation at specific sites or binding of protein domains, to macro steps such as the results of signaling or metabolic modules. The choice of level of detail depends both on available data and the questions to be asked. This flexible approach allows one to study reaction networks of hundreds or even thousands of nodes.

Although detailed data concerning reaction rates is sparse, there is much more data concerning time series and overall effects of changes in cellular signals and expression levels of different cellular components. Thus there is increasing interest in developing simulation and verification tools to handle quantitative or semi-quantitative data. Moreover it is important to address probabilistic aspects of biochemical processes. Thus one would like to ask a model semi-quantitative questions about the possible outcomes under different initial conditions or due 
to perturbations of ongoing processes, without sacrificing the ability to scale to moderately complex processes. For example, one might ask about the relative amounts of different phenotypes related to an overexpressed gene; or how the outcomes change when the network is perturbed, say by mutations or blocking activity of particular components.

In this paper we report on a study of different approaches to represent information about approximate quantities and rates as a first step to extending the Pathway Logic modeling framework. The underlying theme is use of probabilities and stochastic modeling as a flexible technique to account for stochastic features and to incorporate different levels of quantitative information. Questions of interest include: how do different mathematical models represent rates and quantities? What are good choices for modeling randomness in these networks? What simplifications and abstractions are meaningful? Which techniques have efficient implementations that can scale to moderately complex modules?

We considered different but related approaches from the literature to represent probabilities and random events: probabilistic boolean networks, stochastic simulations of chemically reacting systems, and stochastic Petri nets. We reinterpreted and customized these different techniques to the framework of Pathway Logic and several variations of these approaches were implemented and compared. Experiments were carried out using the ErbB dimerization network as a testbed. A first simple approach based on prioritizing rules and using a greedy discrete algorithm for simulation was developed and tested on a model of dimerization and activation all four ErbBs. The greedy algorithm was compared to an analysis using a model-checker for probabilistic systems. The feasibility of predicting the final/equilibrium state for a smaller model of dimerization of two ErbBs was first tested using a probability-based-rule-sampling approach, programmed in Matlab. Then, a probabilistic extension of the rewriting semantics underlying Pathway Logic was implemented in the Maude rewriting logic language and an extension of the small ErbB dimerization network with rules for internalization and degradation of the ErbBs was studied and compared to a previously published model.

\section{Symbolic modeling and Pathway Logic}

This section begins with a brief summary of approaches to modeling biological processes based on symbolic formalisms, then discusses the Pathway Logic approach in more detail.

\subsection{Symbolic modeling of cellular processes}

Symbolic/logical models allow one to represent partial information and to model and analyze systems at multiple levels of detail, depending on information available and questions to be studied. Such models are based on formalisms that provide language for representing system states and mechanisms of change such as reactions, and tools for analysis based on computational or logical inference. 
Symbolic models can be used for simulation of system behavior. In addition properties of processes can be stated in associated logical languages and checked using tools for formal analysis. A variety of formalisms have been used to develop symbolic models of biological systems, including Petri nets $[4,5]$; the picalculus [6] and stochastic variants [7]; stochastic logics and associated model checkers [8]; ambient/membrane calculi [9,10]; statecharts [11]; live sequence charts [12]; and rule-based systems including P-systems [13]; BioCham [14, 15]; and Pathway Logic [1-3]. Each of the underlying formalisms was initially developed to model and analyze computer systems with multiple processes executing concurrently.

\subsection{Pathway Logic}

In Pathway Logic (PL) models of biological processes are developed using the Maude system [16] a formal language and tool set based on rewriting logic. Rewriting logic [17] is a logical formalism that is based on two simple ideas: states of a system are represented as elements of an algebraic data type; and the behavior of a system is given by local transitions between states described by rewrite rules. The process of application of rewrite rules generates computations (also thought of as deductions). In the case of biological processes these correspond to pathways.

A PL model includes representation of cellular components such as proteins and small molecules, their locations, protein state, and post translational modifications. It also includes representations, as rewrite rules, of basic process steps such as metabolic reactions or intra- and inter- cellular signaling. Execution of the rules allow one to represent and reason about dynamic assembly of complexes, cascading transmission of signals, feedback-loops, cross talk between subsystems, and larger pathways. Pathways are not predefined. Instead they are assembled by instantiating and connecting individual steps, starting from an initial state, subject to user-defined constraints. PL models are transformed into Petri nets for visualization and analysis using the Pathway Logic Assistant, a tool for interactive visualization and analysis of PL models.

In the following we use the EGFR family of receptor tyrosine kinases (ErbBs), important in the study of cancer tumor cells, as the basis of our case studies. These receptors form a multiplicity of homo- and hetero-dimers [18]. As an example, the following are rules (in simplified form, represented using Maude syntax) for the homo- and hetero-dimerization of two receptors ErbB1 (also known as EGFR) and ErbB2 (also known as HER2).

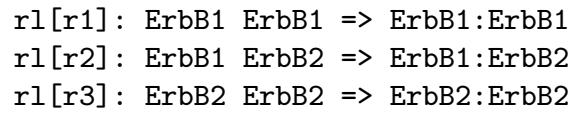

The first rule (labeled r1) says that if ErbB1 is present in the system in multiple copies, then two can bind together to form a homo-dimer ErbB1:ErbB1. When this rule fires two occurrences of ErbB1 are removed from the state and ErbB1:ErbB1 
is added. The second rule describes the hetero-dimerization of ErbB1 and ErbB2, and the third rule describes homo-dimerization of ErbB2.

Sample PL models, tutorial material, papers and presentations are available from the PL web site, http://pl.csl.sri.com/, along with the Pathway Logic Assistant [19].

\section{Prioritized rule modeling of ErbBs}

We started with a very simple idea for semi-quantitative reasoning, namely to assign priorities to rules. The priorities can be thought of as affinities or an abstraction of the thermodynamics. We used these priorities in two ways: as parameters to a greedy algorithm for choosing which rule to fire next; and as parameters of a probabilistic model. This idea was tested on a simplified set of rules for the four members of the ErbB family of receptors. These rules model the (homo- and hetero-) dimerization and resulting cross phosphorylation steps, assuming the receptors that need ligands are initially ligand bound.

$r 1[1]:$ E2 E3B $\Rightarrow$ E3Bp E2d

$r 1[2]: E 2 \quad E 1 B \Rightarrow E 2 p$ E1Bp

$r 1[3]: E 2 \quad E 4 B \Rightarrow E 2 p \quad E 4 B p$

$r l[4]: E 1 B$ E1B $\Rightarrow$ E1Bp E1Bp

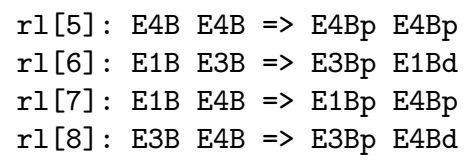

In these rules E1B represents ligand bound ErbB1, and similarly for E3B and E4B. E2 represents ErbB2 which has no ligand. E1Bp is bound phosphorylated ErbB1, implicitly dimerized with its phosphorylation partner, and E1Bd represents ErbB1 that is dimerized but not phosphorylated. Similarly, for the other ErbBs. The rules express the known biochemistry of the ErbB dimers. In particular ErbB3 has no kinase activity and thus can not cross phosphorylate its dimerization partner. Also rules for homo-dimerization of ErbB2 and ErbB3 have been omitted. For simulation purposes an execution state is a set of pairs $(n, e)$, where $e$ is one of the ErbB symbols and $n$ is the number of molecules of $e$.

In the following subsections we explain the two uses of priorities, give results from some test cases, and compare the two methods.

\subsection{Greedy algorithm}

The greedy algorithm for using the priorities is the following: all rules of highest priority are applied until none can be applied. Then rules of the next highest priority are considered, until all of the priority levels are exhausted. Note that because there are no cycles (no rule produces something another rule can use) if a rule is applied as much as possible, application of other rules will not result in a state where the rule is again applicable.

The table below summarizes the results of 4 test cases: two starting states and two assignments of rule priorities. The two starting states are

$((10000 \times$ E1B $)(100000 \times$ E2 $)(100000 \times$ E3B $)(n \times E 4 B))$ 
where $n$ is 0 or 10000 . The first assignment gives all rules have same priority and second assignment is the following

$r l[1] \rightarrow 1, r l[2] \rightarrow 2, r l[3] \rightarrow 2, r l[4] \rightarrow 3$, $r l[5] \rightarrow 3, r l[6] \rightarrow 4, r l[7] \rightarrow 4, r l[8] \rightarrow 4$.

where lower numbers correspond to higher probability, reflecting the experimental observation that ErbB2 is the preferred dimerization partner of the ErbBs.

The row labels code the test case, eq is the same priority case and neq is the varied priority case. The +/- corresponds to presence or absence of ErbB4 in the initial state.

\begin{tabular}{|c|c|c|c|c|c|c|c|}
\hline & E1Bd & E1Bp & E2d & $\mathrm{E} 2 \mathrm{p}$ & E3Bp & $\mathrm{E} 4 \mathrm{Bd}$ & E4Bp \\
\hline eq- & 3334 & 6668 & 96666 & 3334 & 100000 & 0 & \\
\hline $\mathrm{eq}+1$ & 2500 & 7500 & 95000 & 5000 & 100000 & 2500 & 500 \\
\hline neq- & 0 & 10000 & 100000 & 0 & 100000 & 0 & \\
\hline neq + & 0 & 10000 & 100000 & 0 & 100000 & 0 & 100 \\
\hline
\end{tabular}

With equal priority rules the presence of ErbB4 effects the outcome for others by competing with ErbB3 for dimerization resulting in more phosphorylation of other ErbBs. With highest probability assigned to dimerization of ErB2 with ErbB3 this reaction uses all the ErbB2 and ErbB3 and ErbB1 and ErbB4 will dimerize with partners that can cross phosphorylate.

\subsection{Probabilistic Model}

A simple algorithm was used to convert rule priorities into probabilities. Namely, starting with the highest priority, for each group of priorities, we give half of the probability left to that group and divide that amount equally among rules in the group. Thus, for the priority assignment above, we get the following probability assignment

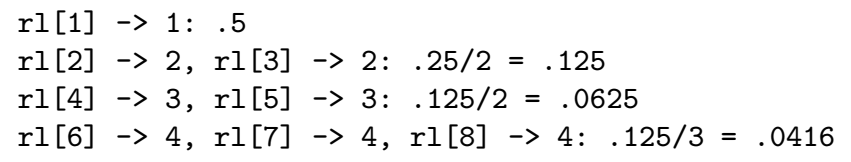

The problem now is, for a given starting state, to determine the probabilities for each of the different ErbBs in the final state. In particular we want the mean of random variables representing the number of different forms of ErbBs. That is we want $E(e)=\Sigma_{p} p \cdot P(|e|=p$ in a final state), where $|e|$ is the quantity of the named ErbB state.

To determine the distribution in a final state we formulated a series of formulas in PCTL about the probability that the value of each random variable is in a given range and used the PRISM model checker [20] to determine the corresponding probabilities. As this is a much more complex process than the discrete case, we used a scaled down version of the initial state for the discrete 
case study with no ErbB4 as a test case: ((10,E1B) (100,E2) (100,E3B)). The table below compares the results using PRISM (row labeled probl) with those using the greedy discrete algorithm (row labeled greedy) to find the distribution in the final state.

$\begin{array}{lclrcrl} & \text { E1BD } & \text { E1BP } & \text { E2D } & \text { E2P } & \text { E3B } & \text { E3BP } \\ \text { probl } & 0.22 & 9.78 & 93.48 & 6.52 & 6.31 & 93.69 \\ \text { greedy } & 0 & 10 & 100 & 0 & 0 & 100\end{array}$

The results turn out to be quite close. We find that the probabilistic computation produces small non-zero values whenever the discrete computation produces zeros. This is consistent with the hypothesis that the probabilistic approach models the stochastic nature of the system. In the case that small non-zero values can safely be ignored, the discrete greedy algorithm is a better choice as it is substantially more efficient. However, if there is a chance that the small amounts could be amplified in a larger context then a probabilistic model is better.

In the following section, we explore more sophisticated choices in extending Pathway Logic to model stochastic behavior. We will analyze the resulting models using stochastic simulation techniques, which fall in between the two extremes of the simplistic "greedy method" and the exhaustive "probabilistic model-checking method" used in this section.

\section{Beyond PL: Quantitative and Probabilistic Modeling}

We extend the PL modeling formalism in two distinct directions: incorporating quantitative information and the notion of time; and incorporating stochastic information into the models. We note here that most stochastic modeling approaches integrate the notion of probability and time and exploit quantitative information to define and handle these concepts. For instance, the classic chemical master equation (CME) [21] describes the time evolution of a probability density function using partial differential equations. Other stochastic modeling formalisms, such as stochastic Petri nets [4], are also given semantics using the CME. As a result, simulation engines for stochastic models produce time series data of species concentrations.

The notion of time elapse and probabilistic transition are inherently coupled. However, as Gillespie points out in his seminal paper [21], while performing a stochastic simulation of CME, there is a certain decoupling between the choice of the next reaction to fire and the time that elapses before the effects of the reaction are observed. This is reflected in Gillespie's Direct Method stochastic simulation algorithm (SSA), where the algorithm samples two distinct random variables for these two purposes.

In our extension of PL, we keep the two aspects separate and make the model modular with respect to the choices for these two parts. As a result, we get a natural and flexible modeling language that is more useful as a modeling and prototyping formalism. We also have the possibility of using different options 
for defining probabilistic rule firing and similarly, different options for specifying the timing behavior.

The syntax of PL changes only very slightly when it is extended with probabilities. With each rule, we now associate a scalar value, called weight or affinity.

$r l[r 1]:$ ErbB1 ErbB1 $\Rightarrow$ ErbB1:ErbB1 a1 $=1$

$r 1[r 2]:$ ErbB1 ErbB2 $\Rightarrow$ ErbB1:ErbB2 a2 $=10$

$r 1[r 3]:$ ErbB2 ErbB2 $\Rightarrow$ ErbB2:ErbB2 a3 $=10$

There are different ways of interpreting these scalar affinities. In any state, the likelihood of a reaction will be proportional to the product of its affinity and the number of each reactant ${ }^{5}$ : given a state $\mathbf{s}$ (represented as a function from species to its number) and a rule $r$ with reactants $r_{1}, r_{2}, \ldots$, let $f_{r}(\mathbf{s})$ denote the product $a_{r} \Pi_{i} \mathbf{s}\left(r_{i}\right)$.

Let Enabled(s) denote the set of all rules that are enabled in the state $\mathbf{s}$, i.e. all the reactions that can possibly occur and change the state of the system. The semantics of PL specifications extended with affinities can be given as a Markov chain. There are different choices for defining such a Markov chain, although in all cases reactions to fire are ultimately chosen by sampling from a uniform distribution. We describe three choices here, exactly one rule, atmost one rule, and multiple rules, that we have explored.

Exactly one rule at a time. One natural way is to assume that in any state, the events set in the probability space consists of all the enabled rules. If we assume that these rules are exclusive-that is no more than one reaction can happen at the same time-and independent, then the probability that a rule $r$ fires in state $\mathbf{s}$ is exactly equal to

$$
\text { Probability of firing } r \text { in state } \mathbf{s} \doteq \frac{f_{r}(\mathbf{s})}{\Sigma_{r^{\prime} \in \operatorname{Enabled}(\mathbf{s})} f_{r^{\prime}}(\mathbf{s})} .
$$

Note that if exactly one rule is enabled in a given state, then that rule is fired with probability 1.

At most one rule at a time. If we assume the existence of a maximum constant $M$ such that $\Sigma_{r \in \operatorname{Enabled}(\mathbf{s})} f_{r}(\boldsymbol{s})<M$ for all states $\boldsymbol{s}$, then the probability that a rule $r$ fires in state $\mathbf{s}$ can be given to be $f_{r}(\mathbf{s}) / M$. In contrast with the previous case, in this case we may have a nonzero probability of no rule firing.

Possible multiple rules. In this case, we allow the rules to fire simultaneously. This is done to account for possible co-occurrence of the rule firing events, as is the case in Probabilistic Boolean Networks. In this case, the event space in state $\mathbf{s}$ consists of $2^{\mid \text {Enabled }(\mathbf{s}) \mid}$ elements: each rule $r \in \operatorname{Enabled}(\mathbf{s})$ may or may not fire. The probability of each event is the product of the probabilities that

\footnotetext{
${ }^{5}$ For completeness, we mention here that the "product of reactants" can be replaced by the more accurate "number of different possible combinations between the reactants" as suggested in [22].
} 
each reaction happens or does not happen in the next time step. Note here the higher computational burden of this method compared to the first two.

The choice of a particular one may depend on the particular network under study, and on the available information (i.e., whether we are given general firing likelihoods, or affinities, or reaction rates).

We have specified above a time-abstract semantics for extended PL specifications. We can incorporate time in the semantics as well. To do this, we need to give a time-dependent interpretation to the affinities. Again, there are a few options here, and we describe two of them below: exponential random and deterministic amortized.

Exponential random variables. While in state $\mathbf{s}$, we assume that the time that elapses before a reaction fires is given by an exponential random variable with decay constant $K \doteq \Sigma_{r \in \text { Enabled }(\mathbf{s})} f_{r}(\mathbf{s})$. In other words, the probability that $t$ time units elapse while in state $\mathbf{s}$ is given by

$$
p_{1}(t \mid \mathbf{s}) \doteq K e^{-K t}
$$

Deterministic amortized variables. We can consider a deterministic approach for computing the time elapse by assuming that the rate of change of a species' concentration is given by the difference between its propensity to be created (by rules that create that species) and its propensity to be destroyed (by rules that use up that species). Given a state $\mathbf{s}$, let $p$ denote a species that is produced in the rules $\operatorname{Prod}(p)$ and consumed in the rules $\operatorname{Cons}(p)$, where $\{\operatorname{Prod}(p), \operatorname{Cons}(p)\} \in$ $\operatorname{Enabled}(\mathbf{s})$. Mathematically, we can say that

$$
d p / d t \doteq \Sigma_{r \in \operatorname{Prod}(p)} f_{r}(\mathbf{s})-\Sigma_{r \in \operatorname{Cons}(p)} f_{r}(\mathbf{s})
$$

Now the time interval $\Delta t$ between two adjacent states, $\mathbf{s}$ and $\mathbf{s}^{\prime}$, from the point of view of species $p$ can be computed by approximating the above expression via a first-order Taylor expansion, and solving for the time step $\Delta t$.

$$
\Delta t=\frac{\left(\mathbf{s}^{\prime}(p)-\mathbf{s}(p)\right)}{\Sigma_{r \in \operatorname{Prod}(p)} f_{r}(\mathbf{s})-\Sigma_{r \in \operatorname{Cons}(p)} f_{r}(\mathbf{s})}
$$

We do not have a notion of global time in this case. Each species has its own clock.

As we mentioned earlier, the explicit decoupling of the state change aspect of a transition (or, equivalently, of the rule selection) from its timing aspects leads to greater flexibility in modeling and simulating PL models with affinities. It allows simulations to be first performed in a time abstract way and then, if required, to embed timing information in the simulation a posteriori, that is after the simulation has been performed.

\section{Other approaches in the literature.}

We review here three related approaches for stochastic modeling that have also partly inspired our choices above. The main difference between PL schemes extended with affinities and the following models is that the extended PL models 
and their simulation engine explicitly decouple time elapse and probabilistic state transition features and allows for more choices in each feature.

The Stochastic Simulation Algorithm. Gillespie's Stochastic Simulation Algorithm (SSA) [21] aims at simulating the evolution of a set of $N$ chemical species interacting through $M$ possible different reactions within a fixed volume $V$. Unlike the classical reaction-rate approach, which sets up a deterministic system of differential equations based on the "law of mass action", Gillespie's algorithm simulates the Chemical Master Equation (CME), which describes both the time and probabilistic transition behavior of the system. Specifically, if $P\left(\mathbf{s}, t \mid \mathbf{s}_{\mathbf{0}}, t_{0}\right)$ denotes the probability that the state is $\mathbf{s}$ at time $t$, given it was $\mathbf{s}_{\mathbf{0}}$ at time $t_{0}$, the CME is given as:

$$
\frac{\partial}{\partial t} P\left(\mathbf{s}, t \mid \mathbf{s}_{\mathbf{0}}, t_{0}\right)=\Sigma_{r}\left[f_{r}\left(\mathbf{s}-\nu_{r}\right) P\left(\mathbf{s}-\nu_{r}, t \mid \mathbf{s}_{\mathbf{0}}, t_{0}\right)-f_{r}(\mathbf{s}) P\left(\mathbf{s}, t \mid \mathbf{s}_{\mathbf{0}}, t_{0}\right)\right]
$$

where $\nu_{r}$ is the vector of the change in the number of molecules of each species caused by a firing of reaction $r$.

Gillespie shows that his stochastic simulation algorithm (SSA) exactly simulates the above chemical master equation, [22]. At each step, the algorithm chooses two quantities:

(i) the time delay $\tau$ for the next reaction to occur;

(ii) the reaction $r$, among the enabled reactions, that will occur next.

The time step $\tau$ is a sample of the exponential random variable with decay constant $\Sigma_{r \in \text { Enabled(s) }} f_{r}(\mathbf{s})$. Note that this is the first option in the two choices for incorporating time in extended PL models described above. The reaction $r$ to fire is chosen by sampling an integer random variable on $[1, M]$ with point probabilities $f_{r}(\mathbf{s}) / \Sigma_{r^{\prime} \in \operatorname{Enabled}(\mathbf{s})} f_{r^{\prime}}(\mathbf{s})$. Note again that this is the first option, among the three options, for defining the next time abstract transition in extended PL models described above. As noted by Gillespie, this particular combination of choices for simulation (and the CME dynamics) is a consequence of the assumption that the propensity function, $f_{r}(\mathbf{s})$ for reaction $r$ in state $\mathbf{s}$ is such that $f_{r}(\mathbf{s}) d t$ defines the probability that the reaction $r$ will fire once in the next infinitesimal time interval $[t, t+d t)$ given that the state is $\mathbf{s}$ at time $t$.

Stochastic Petri Networks. In Section 2.2, we mentioned that a PL model essentially encodes a Petri net. Stochastic Petri nets are an extension of Petri nets that incorporate random events. There are many variants of stochastic Petri nets in the literature. The standard stochastic Petri nets can also be given semantics using a chemical master equation, and hence the stochastic simulation algorithm can be used to perform simulations on such models [4]. The literature on stochastic Petri nets also considers other semantics for time elapse given by non-exponential random variables. Moreover, there are variants, called general stochastic Petri nets, that allow immediate and time delayed transitions. 
Probabilistic Boolean Networks. Probabilistic Boolean Networks (PBN) have been introduced to model and simulate regulatory networks through a rule-based approach [23]. They are described by a set of boolean-valued nodes and functions (rules) that update these nodes. The rules to be fired are chosen probabilistically allowing for possible multiple rule firings. Unlike the Gillespie's Stochastic Simulator, they do not embed any notion of time, but just sequentially execute a particular set of rules.

\section{Implementation and Simulations}

In this Section of the paper we describe the implementation of the ideas and two simulation case studies.

Simple ErbB dimerization network (sErbB): We consider the simple biological system containing three second-order forward reactions first described in Sec. 2.2 and enhanced with the definition of affinities, as in Sec. 4. No reverse reaction is modeled in this simple network. Considering the corresponding reaction equations, the quantitative parameters are the concentrations of each reactant $(E 1 \doteq \operatorname{ErbB} 1$ and $E 2 \doteq$ ErbB2 $)$ and dimerized products $(E 1 E 1 \doteq$ ErbB1:ErbB1, $E 1 E 2 \doteq \mathrm{ErbB} 1:$ ErbB2, $E 2 E 2 \doteq \mathrm{ErbB} 2:$ ErbB2). Associated to each reaction are the three "association affinities" ai.

If we conceive the aforementioned affinities as reaction rates, we can come up with a system of ordinary differential equations (ODEs) for solving the above network kinetics:

$$
\begin{gathered}
\frac{d E 1 E 1}{d t}=\mathrm{a} 1 \cdot E 1 \cdot E 1 ; \quad \frac{d E 1 E 2}{d t}=\mathrm{a} 2 \cdot E 1 \cdot E 2 ; \quad \frac{d E 2 E 2}{d t}=\mathrm{a} 3 \cdot E 2 \cdot E 2 ; \\
\frac{d E 1}{d t}=-2 \mathrm{a} 1 \cdot E 1 E 1-\mathrm{a} 2 \cdot E 1 E 2 ; \quad \frac{d E 2}{d t}=-2 \mathrm{a} 3 \cdot E 2 E 2-\mathrm{a} 2 \cdot E 1 E 2 .
\end{gathered}
$$

Extended ErbB interaction network (eErbB): A more complete ErbB interaction network was constructed based on an earlier work (see [24],[25]) and reported in Appendix 2. The dissociations (reactions 4,5,6) and internalizations (which products are denoted by subscripts in) of the dimerized products are additional reactions in this network, compared to sErbB. Rate constants are named according to [24] and are assumed to be known.

Quantitative and Probabilistic Rule Selection. We implemented a Probabilistic Rewriting Module (PRR) in Maude. The following direct scheme describes the set of Maude procedures that is executed, where meta-level maude programming is used.

1. A multiset of reactants and products with their associated quantities is defined as a "state". 
2. A chemical reaction is represented by a "rule" where a multiset of reactants turn into a multiset of products. Applying a rule yields a decrease of the quantity of reactants and and increase of the quantity of products according to the reaction stoichiometry.

3. The firing likelihood of a rule is computed with a probability that depends on one of the approaches in Sec. 4.

4. At each step of the PRR simulation a rule is selected according to its probability; the selected rule is applied; the state is updated and specific new probabilities are computed. The rule rewriting and the state update are performed in meta level.

5. Simulation terminates when there is no rule that can be selected according to the current state. For a network where no reverse reaction is considered (e.g., sErbB), PRR stops at the end of reactants usage. For network with feedback loops (e.g., eErbB) PRR stops when the steady state is reached, which can be inferred from the stabilization of the quantities of the species.

6. As a post processing step, time information is optionally embedded into the simulation data by using one of the approaches in Sec. 4. Further details can be found in Appendix 1.

\section{Discussion of Results.}

We first analyzed the simple network of growth factor receptor dimerization $s E r b B$ to test the methodology and the performance of the PRR module in predicting the steady state of the biological pathway. We then enabled the time embedding feature (see Sec. 4) to add some dynamics to the simulations. Furthermore, both sequential and dynamic simulations were performed for the extended $e E r b B$ interaction network. As we shall see, the results are in good agreement with experiments and previous computational studies.

\section{Simulating sErbB dimerization by PRR module.}

Comparison between PRR and the deterministic rate equations. The deterministic simulation of the $s E r b B$ network was obtained by solving the corresponding kinetic rate equations (see beginning of Sec. 5) in MATLAB. The PRR procedure was executed via routines written in Maude. The initial concentrations of reactants (ErbB1, ErbB2) and products (the homo-dimers ErbB1:ErbB1, ErbB2:ErbB2 and the hetero-dimer ErbB1:ErbB2), as well as the affinities (a1, a2, a3) were varied systematically to examine the response of the network.

Fig. 1 shows PRR simulations for two of the probability models ${ }^{6}$ (exactly one rule at a time and possible multiple rules). The predictions from the two prob-

\footnotetext{
${ }^{6}$ Concentration of reactants and products has a unit of number-per-volume and a volume 1 is assumed for all reactions studied in this work. The rate constants are the inverse of the concentration, times seconds, for the second-order dimerization reaction, and the inverse of seconds for the first order dissociation or internalization reactions.
} 

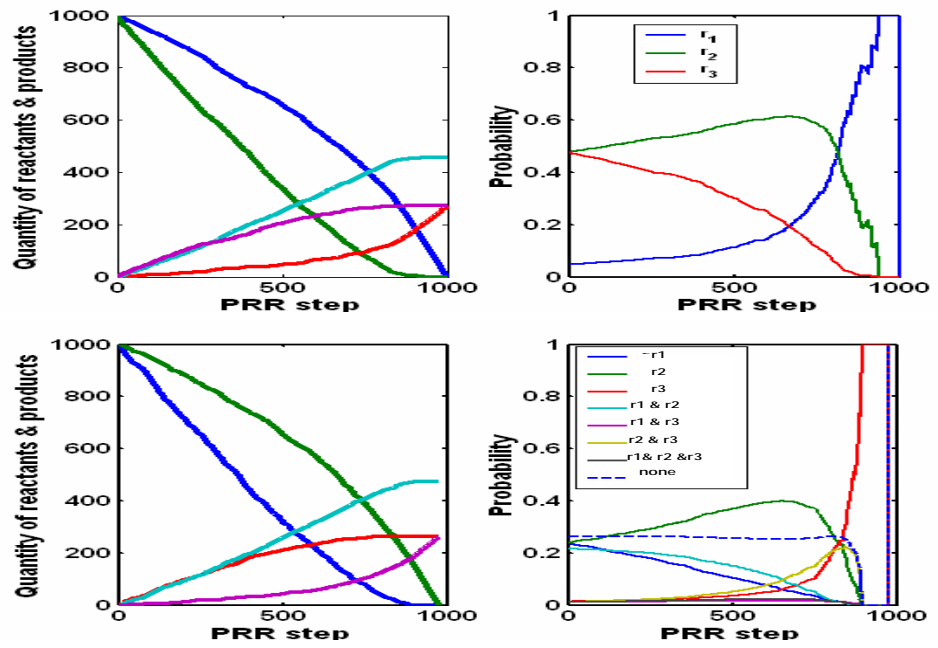

Fig. 1. PRR simulation of $s E r b B$ interaction network (ErbB1: blue, ErbB2: green, ErbB1:ErbB1: red, ErbB1:ErbB2: cyan, ErbB1:ErbB2: magenta) using the single rule (top) and multi-rule (bottom) probability models, given initial state ErbB1 = ErbB2 $=$ 1000, ErbB1:ErbB1 = ErbB1:ErbB2 = ErbB2:ErbB2 =0, and $a 1=1$, a2 $=$ a3 $=10$. The change of quantities of the reactants and products (left plots) and the probabilities of the reactions are plotted along the simulation evolution (right plot).

ability models are similar: the final state contains approximately 500 heterodimers ErbB1:ErbB2 (Fig. 1, left panels, cyan line) and approximately 200 homo-dimers ErbB1:ErbB1 and ErbB2:ErbB2 (Fig. 1, left panels, red and magenta lines). The statistical significance of this similarity was confirmed from 500 independent runs of the simulations. The similar values for the outputs ErbB1:ErbB1 $\approx$ ErbB2:ErbB2 are due to the equality the inputs ErbB1 = ErbB2 and the symmetric topology of the network. The probability values of the reactions in the network are plotted along the steps of PRR simulation for both probability models (Fig. 1, right plot, top for the single-rule model and bottom for multiple-rules model). The last rule selection model illustrates that the concurrency of a set of reactions, as a product of multiple probabilities, is insignificant in general compared to the individual reaction for this particular example, where symmetry and high quantities play an important role. Yet a non-negligible probability of concurrent reactions could occur if the concurrent reactions have considerably higher likelihood than the single reactions, as shown in Fig. 1.

Responses of sErbB network to change of input variables. The response of the $s E r b B$ network to changes on the initial reactants concentration or the affinity of the reactions are explored in Figure 3, in Appendix 1. To summarize the outcomes, the observed non-sensitive response of the network to affinities under the "over expression" condition suggests a possibility to predict a system behavior with incomplete knowledge. In other words, accurate measurements of affinities 
are not strictly discriminating in this case. The less strict requirement of the input parameter would be extremely beneficial in analyzing biological data as experimental accuracy is very limited in in-vivo studies.

Incorporating time into the PRR module. It is possible to enhance the outcomes of the PRR procedure by time-scaling the sequential data: as discussed in Sec. 4 , this can be achieved either by post-simulation Taylor expansion (amortized approach), or by the application of the Gillespie algorithmic idea (exponential assumption). As shown in Fig. 2, plotting PRR prediction against the time by the amortized method (left) or by the exponential embedding (right) yields behaviors that agree with those from the ODE approach, as expected. As the network contains large numbers of reacting molecules (as expected for experimental conditions of the ErbBs dimerization), the time interval between two successive reaction events is small enough to validate the the first order Talyor expansion. Similarly, with these large numbers of reactants, the stochastic simulation (Gillespie algorithm) converges to the ODEs trajectories. Note that the apparent inconsistent termination in the time-resolved kinetics of reactants or products in the traces of ErbB2, ErbB1:ErbB2 and ErbB2:ErbB2 in Fig. 2(left) is explained by our a-posteriori method for embedding time, whereby local clocks for consumed reactants stop. As ErbB2 is consumed faster and is the first to be used up due to the higher dimerization rate, the products (ErbB1:ErbB2, ErbB2:ErbB2) run out accordingly from the reactions that require ErbB2 as reactant. The procedure thus treats this behavior as the termination of the kinetics for ErbB2, ErbB1:ErbB2 and ErbB2:ErbB2.
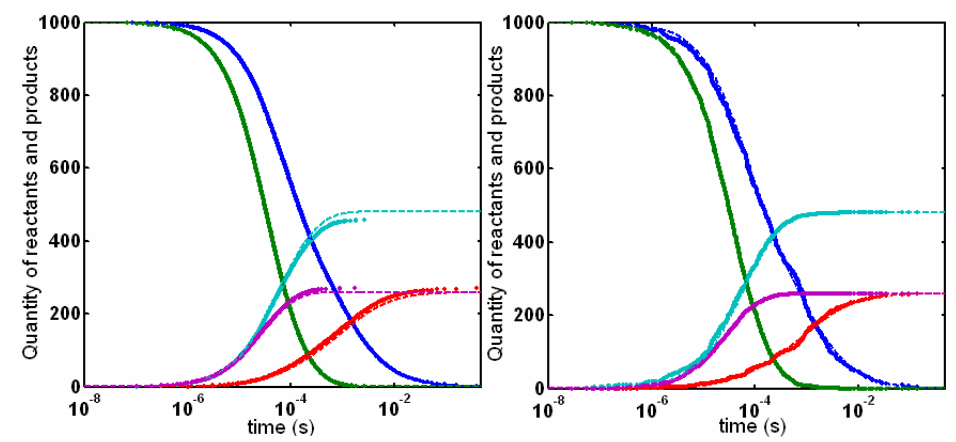

Fig. 2. $s E r b B$ network kinetics (ErbB1: blue, ErbB2: green, ErbB1:ErbB1: red, ErbB1:ErbB2: cyan, ErbB2:ErbB2: magenta) predicted by time-resolved PRR (dots) via Taylor approximation (left) and exponential firing (right), in comparison to ODEs traces (dash lines). Initial state are ErbB1 = ErbB2 = 1000, ErbB1:ErbB1 = ErbB1:ErbB2 = ErbB2:ErbB2 =0, and $a 1=1, a 2=a 3=10$. 
Applying PRR to the more complex eErbB dimerization network. We applied the stochastic simulations to the more complex eErbB biological network which, based on the work in [24], additionally models dissociation of the dimers and internalization of monomers and dimers; both steady state and kinetic studies were performed and compared to the results obtained there; the study of the equilibrium (Appendix 2, Fig. 4, left) has showed, under the substrate saturating conditions, that the relative amount of hetero-(ErbB1:ErbB2) and homo-(ErbB1:ErbB1, ErbB2:ErbB2) dimers varies according to the initial expression levels of ErbB1 and ErbB2 (named, respectively, EGFR and HER2 in [24]).

Four extreme conditions were studied there, corresponding to the four corners of the horizontal plane in Fig. 4 (left): 1. Both ErbB1 and ErbB2 are normally expressed, $3 \cdot 10^{4}$ per cell; 2 . ErbB1 is over-expressed, $6 \cdot 10^{5}$ per cell; $3 . E r b B 2$ is over-expressed, $6 \cdot 10^{5}$ per cell; 4 . Both ErbB1 and ErbB2 are over expressed. We then performed the sequential PRR simulations with similar initializations: 1. $\operatorname{ErbB} 1=\operatorname{ErbB} 2=10^{3} ; 2 . \operatorname{ErbB} 1=2 \cdot 10^{4}$, ErbB2 $=10^{3} ; 3 . \operatorname{ErbB} 1=10^{3}$, $\operatorname{ErbB} 2=2 \cdot 10^{4} ; 4 . \operatorname{ErbB} 1=\operatorname{ErbB} 2=2 \cdot 10^{4}$. Note that we scaled down the absolute quantities of ErbBs in this simulation for coherence with previous sections, but we kept the same ratio of over-expression and normal expression, i.e. 20-fold; in addition, we simplied the network by assuming an EGF saturation condition such that any monomer or dimer species without EGF bound is eliminated. According to these modifications, reproducing exactly the outcome in [24]) with a scaling factor of 20 is not to be precisely expected. Nevertheless, our results shown in the Table below are consistent with the outputs in Appendix 2, Fig. 4, left ${ }^{7}$.

\begin{tabular}{|c|c|c|c|}
\hline ErbB1 & ErbB2 & ErbB1 :ErbB2 - ErbB1 :ErbB1 & ErbB1:ErbB2 - ErbB1:ErbB1 \\
\hline $10^{3}$ & $10^{3}$ & 6 & $7.6 \cdot 10^{3}$ \\
\hline $10^{3}$ & $2 \cdot 10^{4}$ & 260 & $1.5 \cdot 10^{4}$ \\
\hline $2 \cdot 10^{4}$ & $10^{3}$ & -5019 & $-2.3 \cdot 10^{5}$ \\
\hline $2 \cdot 10^{4}$ & $2 \cdot 10^{4}$ & 2971 & $1.6 \cdot 10^{5}$ \\
\hline
\end{tabular}

We also performed kinetic simulations with both ErbBs normally expressed (initial state 1.) and ErbB2 over-expressed (initial condition 2.). Signaling homodimer (ErbB1:ErbB1) and hetero-dimer (ErbB1:ErbB2) were calculated by timestretched PRR simulations (Appendix 2, Fig.5). The outcomes in Fig. 5 under these two circumstances are analogous to those in Fig. 4, right: when ErbB1 and ErbB2 are both $3 \cdot 10^{4}$ (normal expression), a similar, a low output of homodimer(ErbB1:ErbB1) and hetero-dimer (ErbB1:ErbB2) is observed; when ErbB2 is 20-fold overexpressed over ErbB1, the hetero-dimers are greatly enhanced and dominate the signaling species, while the homo-dimers are suppressed down to ground level. The good agreement with [24](Appendix 2, Fig. 4, right) indicates the adequacy of the PRR module in handling these network kinetics.

7 The values of the fourth column are taken from [24] and reported in Appendix 2, Fig. 4, left. 


\section{Conclusion and Future Directions}

We have discussed the underlying principles and several approaches for extending Pathway Logic with the ability to represent and reason about semi-quantitative and probabilistic aspects of biological processes. In summary our conclusions are

- The probability-based rule-selection strategy represents a reasonable approach to incorporate semi-quantitative information into Pathway Logic. Applying this strategy to modeling ErbB dimerization networks shows good agreement with the rate equation approach in predicting the final state.

- A flexible approach to modeling temporal aspects was developed, allowing simulations to be time sensitive, or to account for time a posteriori.

- Traditionally only the evolution of the quantities of reactants are observed when carrying out simulations. We observed that it is also interesting to observe the evolution of reactions probabilities.

- There is a wide range of options to consider when analyzing a model containing some quantitative data. Depending on the number of reactants present, the accuracy of available quantitative information, the question of interest, and the abstraction level, one could use fast greedy approaches, or use more accurate stochastic simulation based approaches with varying degrees of simplifications.

There are a number of interesting questions left for future work. Experiments showed that simulations in which multiple reactions occur simultaneously yield results very close the simulations based on one reaction occurring at each step. This apparently is due to the product of probabilities of the single reactions being too small to have a substantial effect. Using a product to compute the probabilities of multi-reaction steps may not be appropriate as it corresponds to synchronous interaction, while in fact multiple independent reactions occur concurrently and asynchronously. A challenging problem is to develop a theory of truly concurrent probabilistic systems, that accounts for asynchronicity and the transformations of time depending on the view of the system.

The simple and efficient greedy algorithm for simulation based on rule priorities gives surprisingly good results, especially when there are abundant quantities of reactants. A next step is to develop hybrid approaches capable of switching between different simulation methods according to suitable conditions. A possible approach would be to use generalized stochastic Petri nets as a formal representation.

\section{References}

1. Eker, S., Knapp, M., Laderoute, K., Lincoln, P., Meseguer, J., Sonmez, K.: Pathway Logic: Symbolic analysis of biological signaling. In: Proceedings of the Pacific Symposium on Biocomputing. (2002) 400-412

2. Eker, S., Knapp, M., Laderoute, K., Lincoln, P., Talcott, C.: Pathway Logic: Executable models of biological networks. In: Fourth International Workshop on 
Rewriting Logic and Its Applications (WRLA'2002), Pisa, Italy, September 19 21, 2002. Volume 71 of Electronic Notes in Theoretical Computer Science., Elsevier (2002) http://www.elsevier.nl/locate/entcs/volume71.html.

3. Talcott, C., Eker, S., Knapp, M., Lincoln, P., Laderoute, K.: Pathway logic modeling of protein functional domains in signal transduction. In: Proceedings of the Pacific Symposium on Biocomputing. (2004)

4. Goss, P.J., Peccoud, J.: Quantitative modeling of stochastic systems in molecular biology using stochastic Petri nets. Proc. Natl. Acad. Sci. U. S. A. 95 (1998) 6750-6755

5. Oliveira, J.S., Bailey, C.G., Jones-Oliveira, J.B., Dixon, D.A., Gull, D.W., Chandler, M.L.: A computational model for the identification of biochemical pathways in the Krebs cycle. J. Computational Biology 10 (2003) 57-82

6. Regev, A., Silverman, W., Shapiro, E.: Representation and simulation of biochemical processes using the pi-calculus process algebra. In Altman, R.B., Dunker, A.K., Hunter, L., Klein, T.E., eds.: Pacific Symposium on Biocomputing. Volume 6., World Scientific Press (2001) 459-470

7. Priami, C., Regev, A., Shapiro, E., Silverman, W.: Application of a stochastic name-passing calculus to representation and simulation of molecular processes. Information Processing Letters (2001) in press.

8. Calder, M., Vyshemirsky, V., Gilbert, D., Orton, R.: Analysis of signalling pathways using the PRISM model checker. In Plotkin, G., ed.: Proceedings of the Third International Conference on Computational Methods in System Biology (CMSB 2005). (2005)

9. Regev, A., Panina, E., Silverman, W., Cardelli, L., Shaprio, E.: Bioambients: An abstraction for biological compartments (2003) to appear TCS.

10. Nielson, F., Nielson, H.R., Priami, C., Rosa, D.: Control flow analysis for bioambients. In: BioConcur. (2003)

11. Efroni, S., Harel, D., Cohen, I.: Towards rigorous comprehension of biological complexity: Modeling, execution and visualization of thymic t-cell maturation. Genome Research (2003) Special issue on Systems Biology, in press.

12. Kam, N., Harel, D., Kugler, H., Marelly, R., Pnueli, A., Hubbard, J., Stern, M.: Formal modeling of C.elegans development: A scenario-based approach. In: First International Workshop on Computational Methods in Systems Biology. Volume 2602 of Lecture Notes in Computer Science., Springer (2003) 4-20

13. Prez-Jimnez, M., Romero-Campero, F.: Modelling EGFR signalling cascade using continuous membrane systems. In Plotkin, G., ed.: Proceedings of the Third International Conference on Computational Methods in System Biology (CMSB 2005). (2005)

14. Fages, F., Soliman, S., Chabrier-Rivier, N.: Modelling and querying interaction networks in the biochemical abstract machine BIOCHAM. Journal of Biological Physics and Chemistry 4 (2004) 64-73

15. Chabrier-Rivier, N., Chiaverini, M., Danos, V., Fages, F., Schächter, V.: Modeling and querying biomolecular interaction networks. Theoretical Computer Science 351 (2004) 24-44

16. Clavel, M., Durán, F., Eker, S., Lincoln, P., Martí-Oliet, N., Meseguer, J., Talcott, C.L.: The Maude 2.0 system. In Nieuwenhuis, R., ed.: Rewriting Techniques and Applications (RTA 2003). Volume 2706 of Lecture Notes in Computer Science., Springer-Verlag (2003) 76-87

17. Meseguer, J.: Conditional Rewriting Logic as a unified model of concurrency. Theoretical Computer Science 96 (1992) 73-155 
18. Yarden, Y., Sliwkowski, M.X.: Untangling the erbb signalling network. Nat. Rev. Mol. Cel. Biol. 2 (2001) 127-137

19. Talcott, C., Dill, D.L.: The pathway logic assistant. In Plotkin, G., ed.: Third International Workshop on Computational Methods in Systems Biology. (2005) $228-239$

20. Hinton, A., Kwiatkowska, M., Norman, G., Parker, D.: PRISM: A tool for automatic verification of probabilistic systems. In Hermanns, H., Palsberg, J., eds.: 12th International Conference on Tools and Algorithms for the Construction and Analysis of Systems (TACAS06). Volume 3920 of Lecture Notes in Computer Science., Springer (2006) 441-444

21. Gillespie, D.: A general method for numerically simulating the stochastic time evolution of coupled chemical reactions. Computational Physics 22 (1976) 403434

22. Gillespie, D.: Exact stochastic simulation of coupled chemical reactions. Physical Chemistry 81,25 (1977) 2340-2361

23. Shmulevich, I., Dougherty, E.R., Kim, S., Zhang, W.: Probabilistic boolean networks: A rule-based uncertainty model for gene regulatory networks. Bioinformatics 18 (2002) 261-274

24. Hendriks, B., Opresko, L., Wiley, H., Lauffenburger, D.: Quantitative analysis of her2-mediated effects on her2 and epidermal growth factor receptor endocytosis. Biological Chemistry 278, 26 (2003) 23343-23351

25. Hendriks, B., Orr, G., Wells, A., Wiley, H., Lauffenburger, D.: Parsing erk activation reveals quantitatively equivalent contributions from epidermal growth factor receptor and her2 in human mammary epithelial cells. Biological Chemistry 280, 7 (2005) 6167-6169 


\section{Appendix 1: sErbB Network}

Extracting Time Tags in the sErbB Model As mentioned in Sec. 4, introducing quantitative information in the models allows further analysis of its dynamics and the possibility of calculating the actual time flow, as opposed to just keeping track of the sequential operations that are performed. In the case of the $s E r b B$ model, the time interval between two adjacent states (the $i^{t h}$ and $(i+1)^{t h}$ ) of a specific species in the network (say $E 1$ ), that is $\Delta t_{E 1_{i}}=t_{i+1}-t_{i}$, is the time interval when reactant $E 1$ changes from state $E 1_{i}$ to $E 1_{i+1}$; it can be deterministically approximated by the "amortized" approach by discretizing the corresponding ODEs and solving for the time stamps:

$$
\begin{aligned}
\Delta t_{E 1_{i}} & =\left(E 1_{i+1}-E 1_{i}\right) /\left(-2 \mathrm{a} 1 \cdot E 1_{i} \cdot E 1_{i}-\mathrm{a} 2 \cdot E 1_{i} \cdot E 2_{i}\right) ; \\
\Delta t_{E 2_{i}} & =\left(E 2_{i+1}-E 2_{i}\right) /\left(-2 \mathrm{a} 3 \cdot E 2_{i} \cdot E 2_{i}-\mathrm{a} 2 \cdot E 1_{i} \cdot E 2_{i}\right) ; \\
\Delta t_{E 1 E 1_{i}} & =\left(E 1 E 1_{i+1}-E 1 E 1_{i}\right) /\left(\mathrm{a} 1 \cdot E 1_{i} \cdot E 1_{i}\right) ; \\
\Delta t_{E 1 E 2_{i}} & =\left(E 1 E 2_{i+1}-E 1 E 2_{i}\right) /\left(\mathrm{a} 2 \cdot E 1_{i} \cdot E 2_{i}\right) ; \\
\Delta t_{E 2 E 2_{i}} & =\left(E 2 E 2_{i+1}-E 2 E 2_{i}\right) /\left(\mathrm{a} 3 \cdot E 2_{i} \cdot E 2_{i}\right) .
\end{aligned}
$$

Responses of sErbB network to change of input variables studied by PRR. The outcomes of the simulations are reported in Figure 3. If the initial quantity of ErbB1 and ErbB2 are comparable (ErbB1 $\approx$ ErbB2), that is, there is no preferential cellular expression in either of the ErbBs, the final quantities of products largely depends on the value of the affinity (Fig. 3, top). However, when one of the ErbBs is over-expressed, e.g., ErbB2, the influence of the affinities is only moderate or weak. The ErbB2:ErbB2 homo-dimer greatly dominates the final state, regardless of the change in the affinities (Fig. 3, bottom). Furthermore, ErbB1:ErbB1 and ErbB1:ErbB2 have insignificant changes (for instance, the heterodimer ErbB1:ErbB2 varies only 2-fold in Fig. 3, cyan lines).

\section{Appendix 2: eErbB Network}

The Extended eErbB Network. We report the reactions involved in this network ${ }^{8}$ :

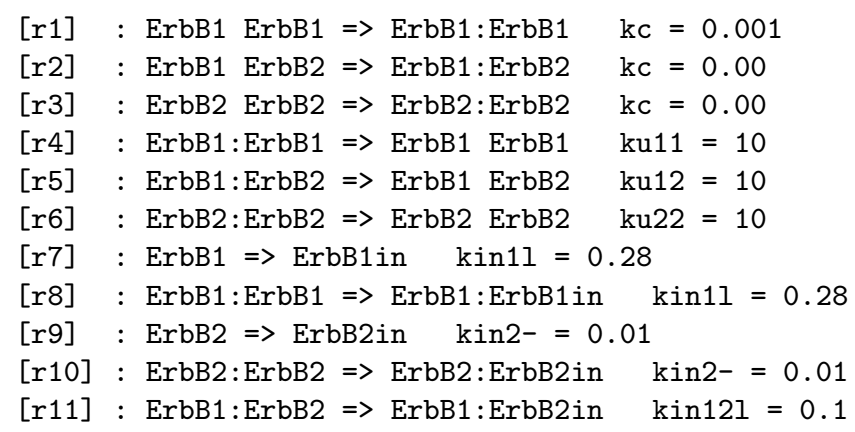

Applying PRR to the more complex eErbB dimerization Network.

$\overline{8}$ The units for $\mathrm{kc}$ are $\left[(\# / \text { cell min })^{-1}\right]$, while the for the other rates are $\left[\mathrm{min}^{-1}\right]$. 

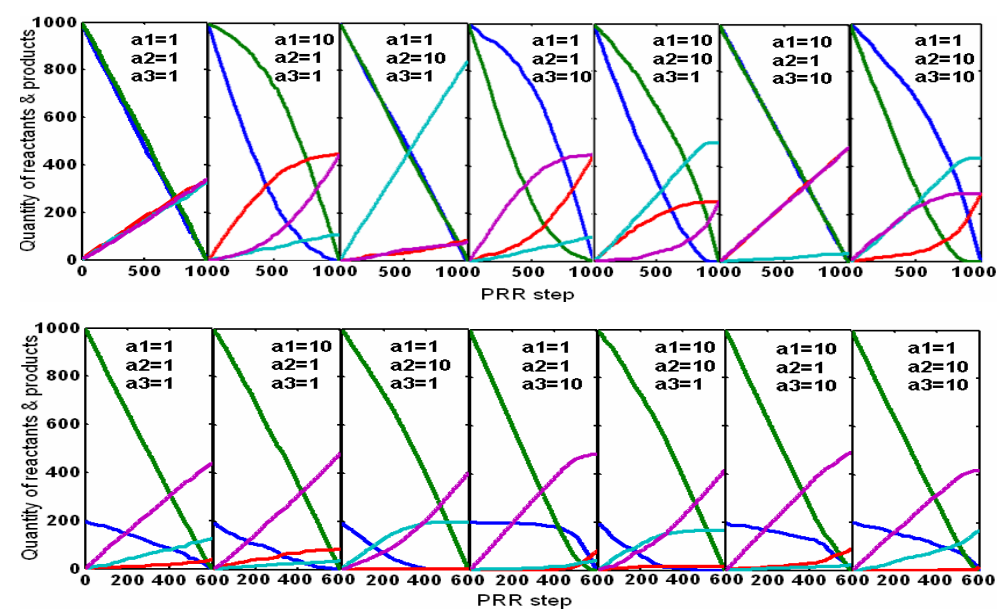

Fig. 3. PRR simulation of sErbB network (ErbB1: blue, ErbB2: green, ErbB1:ErbB1: red, ErbB1:ErbB2: cyan, ErbB2:ErbB2: magenta) with altered reaction affinities. Top: the initial condition is ErbB1 = ErbB2 = 1000, ErbB1:ErbB1 = ErbB1:ErbB2 = ErbB2:ErbB2 $=0$. Bottom: The initial condition is ErbB1 $=200$, ErbB2 $=$ 1000,ErbB1:ErbB1 = ErbB1:ErbB2 = ErbB2:ErbB2 = 0. The affinites are indicated within the single plots.
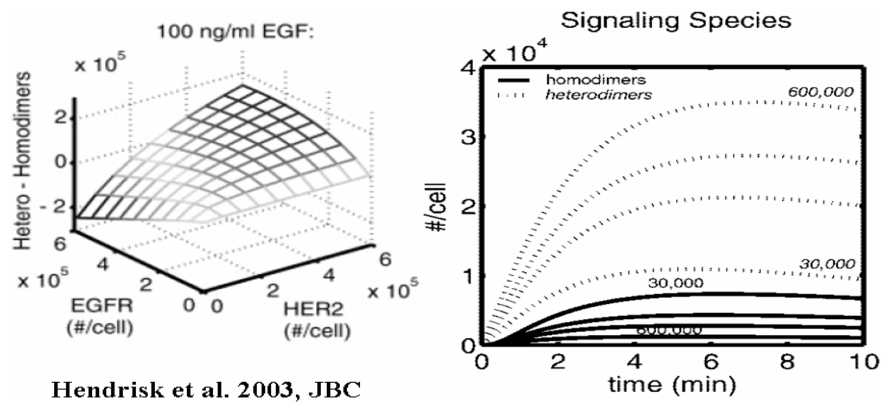

Fig. 4. A steady state simulation and a kinetic study of eErbB presented in [24]. 


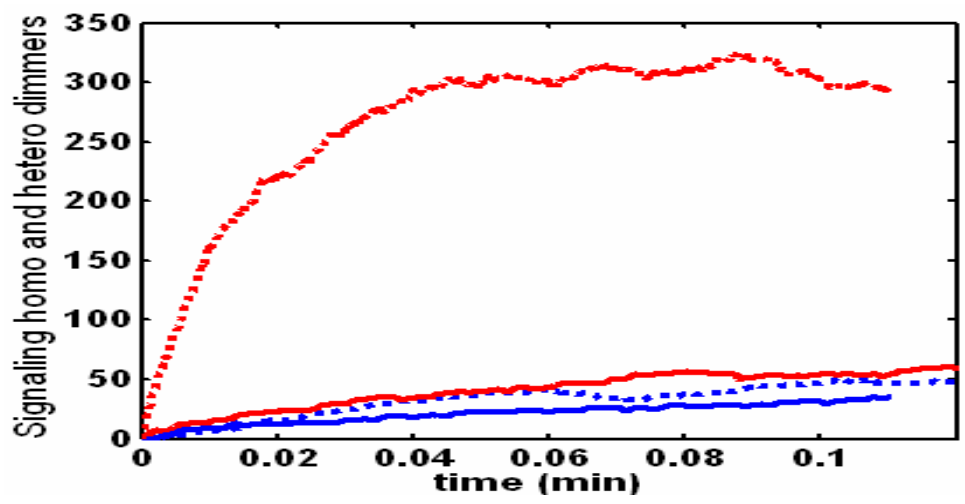

Fig. 5. Quantities of signaling homodimer (ErbB1:ErbB1, blue) and heterodimer (ErbB1:ErbB2, red) under normal expression (initial values ErbB1 $=$ ErbB2 $=1000$, solid lines) or over-expression (initial values ErbB1 $=1000$, ErbB2 $=20,000$, dotted lines) condition, predicted by time-resolved PRR. 